\title{
SILVERMAN Gail P., A woven book of knowledge. Textile iconography of Cuzco, Peru
}

Annabel Vallard

\section{OpenEdition}

\section{Journals}

Édition électronique

URL : https://journals.openedition.org/jsa/12765

DOI : 10.4000/jsa. 12765

ISSN : 1957-7842

\section{Éditeur}

Société des américanistes

\section{Édition imprimée}

Date de publication : 5 décembre 2009

Pagination : 306-312

ISSN : 0037-9174

\section{Référence électronique}

Annabel Vallard, "sitverman Gail P., A woven book of knowledge. Textile iconography of Cuzco, Peru », Journal de la Société des américanistes [En ligne], 95-2 | 2009, mis en ligne le 11 mars 2010, consulté le 04 septembre 2022. URL : http://journals.openedition.org/jsa/12765 ; DOI : https://doi.org/10.4000/ jsa. 12765

Ce document a été généré automatiquement le 4 septembre 2022

Tous droits réservés 


\title{
SILVERMAN Gail P., A woven book of knowledge. Textile iconography of Cuzco, Peru
}

\author{
Annabel Vallard
}

\section{RÉFÉRENCE}

SILVERMAN Gail P., A woven book of knowledge. Textile iconography of Cuzco, Peru, The University of Utah Press, Salt Lake City, 2008, 226 p., bibl., gloss., index, nombr. ill. N\&B, 16 p. de pl. coul., tabl.

1 A woven book of knowledge est un ouvrage doublement attendu de ceux qui s'intéressent aux textiles et à leur iconographie. D'abord, il s'agit de la traduction en anglais de la version révisée de quelques-uns des articles de Gail P. Silverman qui se consacre depuis une vingtaine d'années aux textiles de Cuzco et de sa région et qui étaient connus, jusqu'alors, des seuls hispanophones. Cette publication est ainsi une porte ouverte sur les travaux de cet auteur et permettra de les diffuser auprès d'un public élargi - dont je fais partie - partageant un intérêt pour les textiles, sans être pour autant spécialiste ni des mondes andins, ni plus généralement des mondes latino-américains. Attendu, cet ouvrage l'est également en raison de la place qu'occupent les Andes dans l'imaginaire des amateurs de textiles, qu'ils soient ou non anthropologues, archéologues ou historiens de l'art. L'ancienneté des pièces archéologiques découvertes dans la région, la diversité de leurs homologues contemporaines ainsi que l'extrême sophistication de leurs techniques et de leurs décors contribuent, en effet, à alimenter une passion internationale et une bibliographie abondante.

2 Parmi les recherches publiées en anglais ou en français, Gail P. Silverman évoque, par exemple, celles de Verónica Cereceda (1978), Sophie Desrosiers (1982), Lynn Meisch (1991) ou Ann P. Rowe $(1975 ; 1977)$ et, à travers elles, celles de Raoul d'Harcourt (1934), l'un de leurs plus fameux précurseurs. La riche bibliographie sur laquelle l'auteur s'appuie dans $A$ 
woven book of knowledge rappelle combien, dans le cadre stricto sensu des Andes péruviennes, l'attention des chercheurs s'est principalement focalisée sur les textiles préhispaniques, notamment ceux des cultures Paracas (Anne Paul 1990) et inca (John Murra 1962 ; Ann P. Rowe 1978, 1992, 1997), laissant quelque peu dans l'ombre les textiles modernes et contemporains, à l'exception notable des tapisseries coloniales (Elena Phipps et al. 2004), des costumes de la fin du Xx ${ }^{e}$ siècle (Blenda Femenías 1987) ou encore des tissus de l'île de Taquile (Mary Frame in Rowe 1986; Elayne Zorn 2004) et de Cuzco (Christine et Edward Franquemont in Rowe 1986 ; Ann P. Rowe 1975, 2002). Ceux-ci ont été aussi abordés par le biais des changements sociaux qu'ont connus, ces dernières décennies, les pratiques textiles de la région selon des perspectives ayant trait à l'économie, au genre, au tourisme ou encore aux constructions identitaires locales, régionales et même nationales.

Précisons qu'il n'est pas rare, au sein de cette bibliographie, qu'un même auteur traite, dans des publications parfois distinctes, de périodes historiques très différentes. En ce sens, les recherches sur les textiles ne dérogent donc pas au penchant général des études andines, qui tendent à élaborer leurs modèles sur un axe diachronique plutôt que sur un axe synchronique, et considèrent les sociétés actuelles comme les héritières de leurs illustres prédécesseurs, inca plus particulièrement (Molinié 2003). Si les chercheurs sont peu nombreux à publier à la fois sur les textiles préhispaniques et contemporains (Ann P. Rowe 1975, 1978, 1984, 1992, 1997, 2002 ; Sophie Desrosiers 1982, 1985, 1988, 1998), tous les spécialistes des textiles de la période coloniale, et beaucoup de ceux qui travaillent sur les textiles contemporains, font référence aux textiles incas, même s'ils ne remontent pas aux cultures plus anciennes (Sophie Desrosiers, communication personnelle). Cette tendance est principalement fondée sur le fait que, parallèlement au métier à pédales d'origine européenne, des métiers à tisser du même type que ceux des périodes préhispaniques (permettant par exemple de produire des textiles à quatre lisières) sont toujours utilisés dans ces régions, souvent avec une division des tâches liée au genre (aux hommes les premiers, aux femmes les seconds). Plus encore, alors que des influences extérieures sont visibles dans les matériaux employés et dans certains des motifs réalisés, une continuité existe manifestement dans les types d'armures produites ainsi que dans les façons de les tisser (Desrosiers 1985 ; 1988). Ces comparaisons restent cependant difficiles dans la mesure où les textiles préhispaniques conservés grâce au climat désertique l'ont plutôt été sur la côte, tandis que les textiles contemporains sont presque uniquement produits dans les hautes terres. Du fait de cette différence régionale, il est dès lors encore malaisé de comprendre ce qui était tissé dans les hautes terres avant les Incas, à l'exception des tapisseries dont les décors peuvent être comparés à ceux qui ont été peints sur les céramiques (Sophie Desrosiers, communication personnelle).

Dans A woven book of knowledge, Gail P. Silverman va plus loin que la seule comparaison diachronique et prend comme postulat de départ - j'y reviendrai - que les Q'ero, du fait essentiellement de leur isolement géographique, «ont davantage conservé le mode de vie des Incas que leurs voisins métis [mestizo]» (p.3). Si l'auteur propose de démontrer que l'iconographie textile contemporaine de la région de Cuzco fonctionne comme une écriture non alphabétique, c'est donc toujours en référence au monde inca préhispanique sur lequel les Q'ero sont supposés ouvrir une fenêtre. Pour étayer son hypothèse, Gail P. Silverman a, tout au long de ses années de terrain, non seulement procédé à un recueil extensif des motifs tissés par les Q'ero, mais les a "lus " à l'aide d'une approche méthodologique conjuguant apprentissage du tissage et enquête terminologique (en quechua) auprès des tisseuses et villageois, cela à l'intérieur et hors contexte des 
pratiques textiles. Elle a également demandé aux Q'ero de dessiner leurs villages, leurs champs, l'espace céleste ou encore les temps saisonniers afin de comparer ces représentations graphiques avec les décors tissés.

Il résulte de cette étude un ouvrage richement illustré en neuf chapitres commençant par une présentation générale des Q'ero (chap.I) et une description de leurs matériaux, techniques et productions textiles (chap. II) et se poursuivant par une analyse des motifs et de leurs significations. Gail P. Silverman propose surtout un décryptage des divers losanges quadripartites très largement représentés dans le corpus textile q'ero et des éléments minimaux les composant (lignes, triangles isocèles, croix, etc.). Elle montre ainsi comment ils figurent des concepts cosmologiques et spatiaux (chap. III), ainsi que les temps quotidiens (chap.IV) et saisonniers (chap.V). Elle se penche ensuite sur la classification des couleurs utilisées dans le champ textile et qui, selon elle, servent, à l'instar des quipu incas, à représenter des biens spécifiques comme des animaux ou de la nourriture (chap.VI), puis sur la représentation graphique du mythe de l'Incarri (chap. VII). Les derniers chapitres de l'ouvrage sont consacrés au lexique graphique des motifs q'ero représentés sous forme de diagrammes accompagnés de leur appellation et du champ de leur signification sémantique (représentation du temps ou de l'espace) (chap. VIII) ainsi qu'à un retour sur les principales conclusions de l'auteur (chap. IX).

6 La proposition de Gail P. Silverman de considérer les textiles non seulement comme un langage (c'est-à-dire avec des règles à décoder), doté en somme d'un vocabulaire, d'une grammaire et/ou d'une syntaxe, mais comme une écriture est très intéressante, notamment parce que d'autres chercheurs la considèrent plutôt comme un langage visuel (Cereceda 1978; Zorn 2004) et que, dès lors, un dialogue théorique se dessine comme perspective d'étude comparative dans le futur. Néanmoins, A woven book of knowledge présente, selon moi, deux limites épistémologiques et méthodologiques qu'il est nécessaire de préciser.

7 D'une part, il me semble difficile d'aborder le monde q'ero contemporain sans préciser la place que ce groupe ethnique occupe aujourd'hui dans l'imaginaire régional et national péruvien. En effet, comme l'a montré Yann Le Borgne (2003), la première expédition ethnographique consacrée aux Q'ero menée en 1955 par l'indigéniste Óscar Núñez del Prado «transforma le Q'ero en figure éponyme de l'authenticité recherchée pour construire le Pérou à venir» (in Le Borgne 2003, p. 143). Depuis lors, les Q'ero sont identifiés comme les derniers Incas. Cette figure mythique du Q'ero représentant «Lo andino ", c'est-à-dire ce qui était, pour les indigénistes, propre aux Andes, aux Incas et par extension à Cuzco, a d'abord été à usage politique et s'est construite en opposition avec celle de criollo liée à la côte et à Lima (ibid., p. 144). Fondée notamment sur le mythe d'Inkarri (auquel Gail P. Silverman consacre un chapitre de son ouvrage), cette figure mythique s'exprime depuis une vingtaine d'années dans le domaine rituel. Les Q'ero sont ainsi de nos jours considérés par les Cuzquéniens comme des spécialistes de la divination et de l'offrande à la Terre mère et aux montagnes sacrées (ibid., p. 153). À ce titre, ils exercent en milieu urbain où ils sont très appréciés. En retour et du fait de leur inscription au cœur de la mystique globale du New Age (Galinier et Molinié 2006), ils font l'objet d'un tourisme ésotérique. Cette double combinaison influence la perception que certains Q'ero - comme ceux de Hatun Q'ero, siège de la quasi-totalité des études qui leur sont consacrées et des voyages touristiques ésotériques - ont désormais des caractéristiques de leur identité qu'ils lient à leur statut de dépositaires d'une tradition incaïque réinventée (Le Borgne 2003, pp. 156-157). L'ouvrage de Silverman aurait gagné 
en force si l'auteur avait évoqué cette affiliation fictive des Q'ero avec les Incas - qu'elle soit apposée et/ou revendiquée - et si, en ne la tenant dès lors pas pour réelle, elle avait discuté de manière critique les terminologies textiles recueillies sur le terrain, terminologies qui font justement référence, selon l'auteur, à des concepts préhispaniques et plus particulièrement incaïques.

Il me semble, d'autre part, qu'une étude sur les textiles et leur iconographie ne peut faire l'économie d'une recontextualisation élargie et ne peut se résumer aux seuls entretiens, recueils de terminologies et dessins réalisés sur demande comme si les motifs étaient signifiants par eux-mêmes en dehors de leur mobilisation humaine effective. Si un langage textile existe, je ne pense pas qu'il puisse se résumer à une iconographie de surface. Il s'ancre dans des épaisseurs textiles, épaisseurs tout à la fois matérielles et sociologiques. Or ces épaisseurs naissent des relations qui se nouent entre les humains qui les fabriquent, les portent, les imaginent, et dans les rapports intimes quotidiennement renouvelés que ces derniers entretiennent avec le tissu. Formée aux techniques de tissage et investie depuis de longues années sur le terrain q'ero, il est dès lors étonnant que Gail P. Silverman n'insiste pas plus dans son ouvrage sur les contextes de production textile et, plus encore, sur les usages des étoffes réalisées. Elle ne donne en effet pas la mesure de l'imbrication étroite que les individus entretiennent localement avec le tissu, imbrication qui aurait sans doute éclairé cet imaginaire qu'ils projettent sur eux et expriment notamment, mais pas seulement, par le biais de leur iconographie.

Malgré ces limites, A woven book of knowledge est une entrée richement illustrée dans le monde jusqu'alors peu connu, pour les non-hispanophones, des textiles q'ero. Ce volume rejoint le très bien documenté et très beau catalogue d'exposition que le Musée des textiles de Washington DC leur a consacré (Rowe et Cohen 2002). Ce dernier en propose une approche très différente qui, sur de nombreux points, contredit les conclusions de $A$ woven book of knowledge. Néanmoins, la déconstruction en autant d'unités minimales des motifs étudiés réalisée par Gail P. Silverman - notamment celle des losanges quadripartites - tout comme les représentations graphiques qu'elle leur consacre fait de son ouvrage un objet d'intérêt pour ceux qui s'appliquent, ici ou ailleurs, à la lecture des iconographies textiles. Ayant d'ores et déjà inspiré des chercheurs hispanophones (par exemple Molinié 1993-1994), sa traduction en anglais servira sans aucun doute, comme l'auteur l'appelle de ses vœux, de point de départ à de nouvelles études sur le sujet.

\section{BIBLIOGRAPHIE}

\section{CERECEDA Verónica}

1978 « Sémiologie des tissus andins : les “Talega” d'Isluga ", Annales : Économies, Sociétés, Civilisations, 33 (5-6), pp. 1017-1035.

DESROSIERS Sophie

1982 Métier à tisser et vêtements andins ou le tissu comme être vivant, CETECLAM, Paris. 
1985 « Une expérience de technologie : la reconstruction d'une ceinture précolombienne à partir d'un texte codé du XVII ${ }^{\mathrm{e}}$ siècle ", Techniques et culture, 6, pp. 111-144.

1988 « Les techniques de tissage ont-elles un sens ? Une proposition de lecture des tissus andins ", Techniques et culture, 12, pp. 31-56.

1998 « Textes techniques, savoir-faire et messages codés dans les textiles des Andes », Techniques et culture, 29, pp. 155-173.

FEMENÍAs Blenda (éd.)

1987 Andean aesthetics: textiles of Peru and Bolivia, Exhibition Catalogue, Elvehjem Museum/ University of Wisconsin, Madison.

GALINIER Jacques et Antoinette MOLINIÉ

2006 Les néo-indiens. Une religion du III millénaire, Odile Jacob, Paris.

HARCOURT Raoul d'

1934 Les textiles anciens du Pérou et leurs techniques, Édition d'Art et d'Histoire, Paris [réédition : Flammarion/musée du quai Branly, Paris, 2008].

LE BORGNE Yann

2003 «Évolution de l'indigénisme dans la société péruvienne. Le traitement du groupe ethnique q'ero ", Ethnographies du Cuzco, Ateliers du LESC, 25, pp. 141-159.

MEISCH Lynn

1991 «We are sons of Atahualpa and we will win : traditional dress in Otavalo and Saraguro, Ecuador ", in Margot B. Schevill, Janet C. Berlo et Edward B. Dwyer (éd.), Textile traditions of Mesoamerica and the Andes: an anthology, Garland Publishing, New York, pp. 145-177.

MOLINIÉ Antoinette

1993-1994 « Héros mythique, dieu étatique : soleil aquatique », Bulletin de la Société suisse des américanistes, 57-58, pp. 25-36

2003 «Introduction », Ethnographies du Cuzco, Ateliers du LESC, 25, pp. 7-15.

MURRA John V.

1962 «Cloth and its functions in the Inca state », American Anthropologist, 64 (4), pp. 710-728.

PAUL Anne

1990 « The use of color in Paracas-Necropolis fabrics: what does it reveal about the organization of dyeing and designing ? ", National Geographic Research, 6 (1), pp. 7-21 [partiellement traduit et republié en 2008 dans le catalogue Paracas, trésors inédits du Pérou ancien, coédition musée du quai Branly/Flammarion, Paris, pp. 58-64].

PHIPPS Elena, Johanna HECHT et Cristina ESTERAS MARTíN (éds)

2004 The colonial Andes: tapestries and silverwork, 1530-1830, Metropolitan Museum of Art/Yale University Press, New York/New Haven.

\section{Rowe Ann Pollard}

1975 «Weaving processes in the Cuzco area of Peru », Textile Museum Journal, 4 (2), pp. 30-46.

1977 Warp-patterned weaves of the Andes, The Textile Museum, Washington, DC.

1978 « Technical features of Inca tapestry tunics », Textile Museum Journal, 17, pp. 5-28.

1984 Costumes and featherwork of the Lords of Chimor. Textiles from Peru's North Coast, The Textile Museum, Washington DC.

1992 « Provincial Inca tunics of the South Coast of Peru », Textile Museum Journal, 31, pp. 5-52. 
1997 « Inca weaving and costume », Textile Museum Journal, 34-35, pp. 5-54.

Rowe Ann Pollard (éd.)

1986 Junius B. Bird conference on Andean textiles, The Textile Museum, Washington, DC.

RowE Ann Pollard et John coHEN

2002 Hidden threads of Peru. Q'ero textiles, Merrell/The Textile Museum, Londres/Washington, DC.

zORN Elayne

2004 Weaving a future: tourism, cloth, and culture on an Andean Island, University of Iowa Press, Iowa City.

\section{AUTEURS}

ANNABEL VALLARD

Docteur en ethnologie. Laboratoire d'ethnologie et de sociologie comparative 